\title{
Elliptical Galaxies: Darkly Cloaked or Scantily Clad?
}

\author{
A. J. Romanowsky ${ }^{1}$, N. G. Douglas ${ }^{2}$, K. Kuijken ${ }^{2,3}$, M. R. Merrifield ${ }^{1}$, \\ M. Arnaboldi ${ }^{4}$, N. R. Napolitano ${ }^{2}$, H. Merrett ${ }^{1}$, M. Capaccioli ${ }^{5}$, \\ K. C. Freeman ${ }^{6}$, O. Gerhard ${ }^{7}$ \\ ${ }^{1}$ School of Physics and Astronomy, University of Nottingham; ${ }^{2}$ Kapteyn \\ Institute, Groningen; ${ }^{3}$ Leiden Observatory; ${ }^{4}$ INAF-Osservatorio \\ Astronomico di Pino Torinese, Turin; ${ }^{5}$ INAF-Osservatorio \\ Astronomico di Capodimonte, Naples; ${ }^{6}$ RSAA, Mt. Stromlo and Siding \\ Spring Observatories; ${ }^{7}$ Astronomisches Institut, Universität Basel
}

\begin{abstract}
Planetary nebulae (PNe) may be the most promising tracers in the halos of early-type galaxies. We have used multi-object spectrographs on the WHT and the VLT, and the new Planetary Nebula Spectrograph on the WHT, to obtain hundreds of PN velocities in a small sample of nearby galaxies. These ellipticals show weak halo rotation, which may be consistent with ab initio models of galaxy formation, but not with more detailed major merger simulations. The galaxies near $L^{*}$ show evidence of a universal declining velocity dispersion profile, and dynamical models indicate the presence of little dark matter within $5 R_{\text {eff }}$-implying halos either not as massive or not as centrally concentrated as CDM predicts.
\end{abstract}

\section{Introduction}

Dark matter has long been inferred around spiral galaxies from their flat HI rotation curves; however, in early-type galaxies (ellipticals and S0s) we do not have this luxury, and progress in finding their total masses has been slow. So not only has a fundamental component of the CDM paradigm remained largely unverified - that there should be similarly extended, massive dark halos around ellipticals - but predictions about the detailed halo properties have not been testable (cf. the halo core issues in late-type galaxies discussed at length in this volume). There is actually an advantage to studying elliptical halos: if one can observe at comparable physical radii, the compact nature of ellipticals implies that this is in a more dark-matter dominated regime than in spirals. Thus messy baryonic physics should have been less important, and it may be easier to disentangle the luminous and dark components' relative mass contributions.

The nominal tracer in elliptical halos is their integrated stellar light. To adequately constrain their dynamics, kinematical measurements must be of sufficient quality to obtain such higher-order moments as the Gauss-Hermite $h_{4}$. But the drop-off in surface brightness makes this approach nonviable outside an elliptical's central parts. The best survey so far is of 21 bright, round ellipticals (O. Gerhard, this meeting), which found the circular velocity profiles $v_{c}(r)$ 
to be roughly constant out to $1-2 R_{\text {eff }}(5-10 \mathrm{kpc})$, and ruled out a constant mass-to-light ratio $(M / L)$ for 3 of the galaxies.

Alternative probes of elliptical halos include globular clusters (GCs), which are handy as bright objects spread out to larger radii than the galaxy light (Côté et al. 2001, 2003) - although they are a disjoint population with different properties to the galaxy. $X$-ray emission from thermalized hot gas filling the halo potential is also useful (D. Buote, this volume); but because the total emission correlates strongly with optical luminosity $\left(L_{\mathrm{X}} \propto L_{B}^{2-3}\right.$; O'Sullivan et al. 2003), and only the brightest sources are within easy reach of X-ray telescopes, the findings are biased toward the more massive systems. Similarly, strong gravitational lenses can also be used to probe into galaxy halos (P. Schneider, this meeting), but any galaxies with massive, centrally-concentrated halos are systematically most likely to be detected as lenses. Rings and disks of $\mathrm{HI}$ and $\mathrm{H} \alpha$-emitting gas are also sometimes found at large radii (M. Arnaboldi et al., this volume); but these are rare, and they may not trace a typical population of ellipticals. Satellite kinematics (e.g., Prada et al. 2003) and weak gravitational lensing (e.g., H. Hoekstra, this volume) can probe the outermost parts of galaxies, but their constraints are statistical: they provide limited information about mass variations with radius and with other galaxy properties.

Planetary nebulae (PNe) are arguably the ideal probes because of their simple connection to the main stellar population of the galaxy (e.g., Peng et al. 2002). Also, they are not affected by any mass-dust degeneracy (Baes \& Dejonghe 2001), and their $5007 \AA$ emission lines readily provide precise velocities.

Various studies with the above methods have found dark matter around individual elliptical galaxies, but as discussed, the selection effects may be severe. Weak lensing and satellite studies also imply massive halos around typical $L^{*}$ ellipticals, but further cross-checks are needed. Indeed, dynamical studies have suggested that some galaxies are much less dark matter-dominated than others (Bertin et al. 1994; Gerhard et al. 2001; N. Napolitano et al., this volume).

Besides searching for dark matter, by probing into elliptical halos we can also test other key properties against predictions of galaxy formation models. These include angular momentum and the distribution of stellar and GC orbits. In the rest of this paper, we present PN kinematical studies of five elliptical galaxies, and some implications for galaxy structure and formation.

\section{PN Kinematics of M87 and M49: Virgo siblings}

The two brightest ellipticals in the Virgo Cluster M87 (=NGC 4486) and M49 (=NGC 4472), had their extensive GC systems studied kinematically (e.g., Côté et al. 2001, 2003). Both these and X-ray studies (Schindler et al. 1999; Matsushita et al. 2002) found massive dark halos. Yet no stellar or PN kinematical studies have been done in these galaxies' halos for comparison.

We have pursued kinematic follow-up studies of the large PN samples discovered around these two galaxies with narrow-band imaging surveys (Ciardullo et al. 1998; X. Hui et al., unpublished). PN kinematic studies have till now been sporadically fruitful because of the low efficiency of spectroscopic follow-up, which seems to be largely due to astrometric difficulties. We used the WYFFOS/AF2 multi-fiber spectrograph on the 4.2-m William Herschel Telescope 
(WHT) on La Palma in May 2000 to observe PNe in M49, giving special attention to the astrometric solution. Even with the 2.7 diameter fibers, it appeared that our recovery rate was not optimal, and we found velocities for $\sim 25$ objects.

As a solution to these difficulties, we devised a new technique, masked counter-dispersed imaging, which is a combination of traditional multi-slit spectroscopy and slitless counter-dispersed imaging (see next section). Using the FORS2+MXU spectrograph at the ESO 8-m UT2 telescope in April 2001, we cut standard masks but with the slit sizes increased to $4^{\prime \prime} \times 4^{\prime \prime}$, ensuring object recovery. We took two complementary images of each field with the dispersion direction reversed, allowing us to cancel out the astrometric errors and derive the velocities to within $\sim 15 \mathrm{~km} \mathrm{~s}^{-1}$. Completing this program for M87, we found $\sim 200 \mathrm{PN}$ velocities; for M49, clouds limited our return to $\sim 100 \mathrm{PNe}$.

\section{PN Spectrograph survey: first results}

To overcome the obstacles to observing extragalactic PN kinematics, our team commissioned a special-purpose instrument, the Planetary Nebula Spectrograph (PN.S) at the WHT (Douglas et al. 2002 and this volume). Using a slitless spectroscopy technique termed counter-dispersed imaging, the efficiency of the PN.S has resulted in a breakthrough in this field: hundreds of PN velocities can be obtained in a single night's observing of a galaxy at a distance of $15 \mathrm{Mpc}$.

The primary program of the PN.S is to survey a dozen bright $\left(m_{B} \leq 12\right)$, round (E0-E2), nearby $(D \lesssim 20 \mathrm{Mpc}$ ) ellipticals, obtaining 100-400 PN velocities in each. Based on the statistical distribution of galaxy shapes (Lambas et al. 1992), we estimate that by selecting for projected axis ratios $q \gtrsim 0.8,75 \%$ of our sample will have intrinsic gravitational potential ellipticities $\epsilon_{\Phi} \lesssim 0.1$ and thus can be well characterized by spherical dynamical models. Other than the constraints above, our sample is designed to encompass a broad spectrum of elliptical galaxies, with a large range of stellar light parameters (luminosity, concentration, shape), rotational importance, and environment.

Our program has so far been beset by bad weather, but we have obtained extensive data on the galaxies NGC 821, NGC 3379, and NGC 4494. For each we have an initial data set of $\sim 100 \mathrm{PN}$ velocities out to $\sim 5 R_{\mathrm{eff}}$, but with additional data reduction we expect these to increase to $\sim 200$ each.

\section{Angular Momentum: weak rotation}

With substantial PN data sets for five galaxies, we can first look at their rotational characteristics. It has long been apparent that the central parts of ellipticals are much less rotationally-dominated than are spirals (e.g., Fall 1983), but there may be large amounts of angular momentum stored in ellipticals' unobserved outer parts - perhaps even more than in spirals because of more dominant major merger histories (J. Primack, this volume). Such high outer rotation is seen in the GCs around M87, but it's not clear that these trace the properties of the dark matter and the main stellar population.

None of the galaxies shows a strong increase in rotation with radius (note though that our sample roundness criterion may introduce a bias toward low spins). Within $\sim 3-5 R_{\text {eff }}$, they have spin parameters $\lambda^{\prime} \sim 0.03-0.07$, and at 

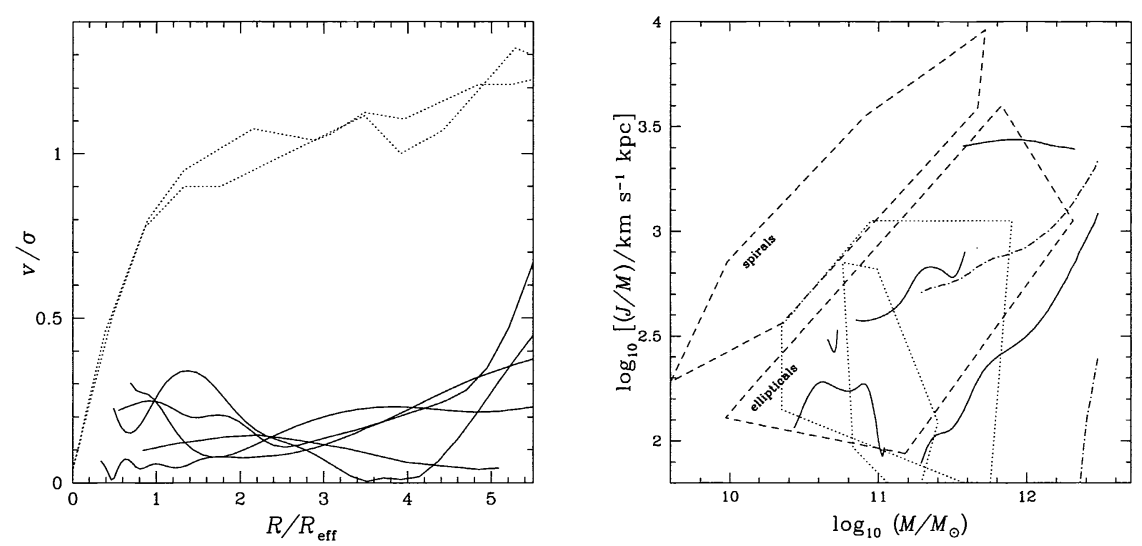

Figure 1. Left: Rotational parameter as a function of projected radius for five galaxies observed with PNe (solid lines), and for a typical simulated merger remnant (dotted lines; Weil \& Hernquist 1996). Right: Specific angular momentum as a function of enclosed mass, after Fall (1983). Dashed lines outline observations for spiral disks and elliptical's central parts. Dotted lines show CDM hydrodynamical simulations for stellar components (Navarro et al. 1995; Eke et al. 2000). Solid lines show a run with radius for the PN galaxies, where the 1- $\sigma$ uncertainties are illustrated for one case by dot-dashed lines.

these radii rotation appears dynamically unimportant: $v / \sigma \sim 0.1-0.3$. This conflicts with major merger simulations that predict rapid outer rotation: $v / \sigma \gtrsim 1$ outside $2 R_{\text {eff }}$ (see Fig. 1, left; Weil \& Hernquist 1996; Bendo \& Barnes 2000).

The ideal comparison is to simulations of galaxy formation in the full cosmological context, but treatments of key baryonic processes such as star formation and feedback are still maturing. Current CDM models do produce low specific angular momenta similar to our observed ellipticals (see Fig. 1, right).

\section{Mass Content: skimpy halos}

Around M87 and M49, we find velocity dispersion profiles that rise slightly with radius, suggesting massive extended dark halos, as found by the GC and X-ray studies. Combining all these constraints together will give us a detailed picture of the mass and orbit distributions. It is initially evident that the stellar orbit radial anisotropy increases into the halo.

The more ordinary $\left(\sim L^{*}\right)$ ellipticals from our PN.S studies, as well as NGC 4697 (Méndez et al. 2001), show something entirely different: their velocity dispersions decline rapidly with radius (see Fig. 2, left). Simple Jeans models with a moderate degree of anisotropy indicate total masses consistent with the visible stars only: a benchmark parameter $\Upsilon_{B 5}\left(M / L\right.$ inside $\left.5 R_{\text {eff }}\right)$ is $\sim 6-15$ (Romanowsky et al. 2003), while stellar populations should have $\Upsilon_{B} \sim 3-12$ (Gerhard et al. 2001). For NGC 3379, we have constructed more versatile orbit 

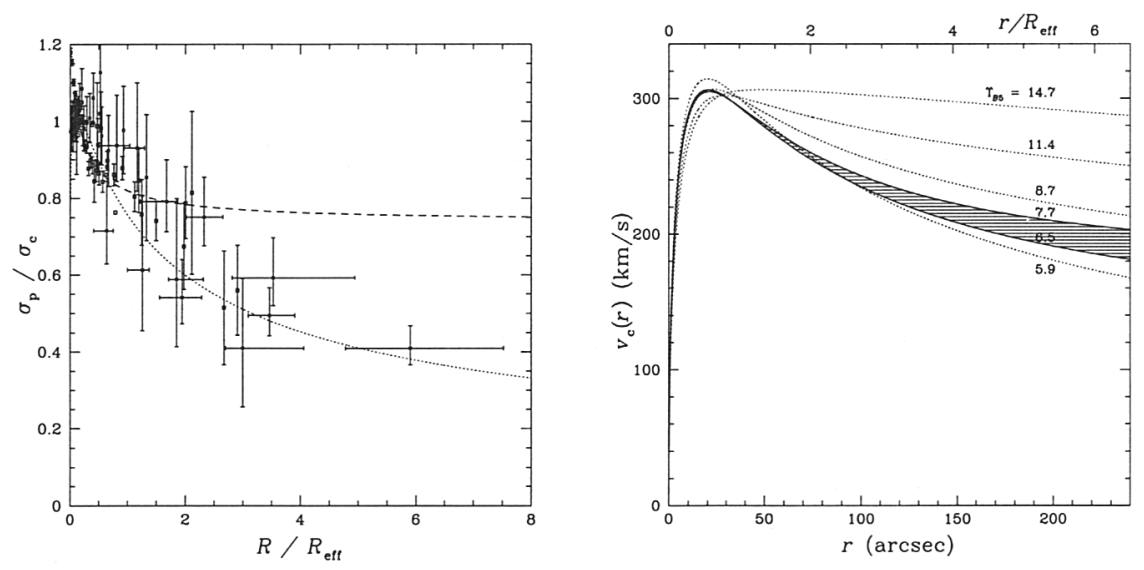

Figure 2. Left: Projected velocity dispersion profiles for four elliptical galaxies (see text) scaled and stacked, as a function of radius, in units of $R_{\text {eff }}$. Open points show PN data; solid points show long-slit stellar data. Simple model predictions are shown for comparison: a singular isothermal halo (dashed line) and a constant- $M / L$ galaxy (dotted line). Right: Circular velocity profile of NGC 3379. The solid lines and shaded area show the region permitted by orbit modeling within the $68 \%$ confidence limits. Dotted lines show excluded models (bottom: constant- $M / L$; top three: more dominant dark halos).

models to allow for the infamous mass-anisotropy degeneracy, and to extract as much information as possible out of the discrete PN velocity data (Romanowsky \& Kochanek 2001). These tightly constrain $\Upsilon_{B 5}$ to be $7.1 \pm 0.6$ (Fig. 2, right). There are still systematic uncertainties in this study, notably the possibility that the galaxy contains a component that is flattened along the line-of-sight; however, independent confirmation of a low $M / L$ comes from an HI ring (Schneider et al. 1989), and from a steeply declining dispersion in the GCs (Beasley et al. 2004; Bergond et al. 2004) - which are unlikely to reside in a flattened system.

For comparison, other results from brighter galaxies typically show $\Upsilon_{B 5} \sim 20$ 40 (Bahcall et al. 1995; Loewenstein \& White 1999). Weak lensing estimates for $L^{*}$ ellipticals, extrapolated inwards in radius, give $\Upsilon_{B 5} \sim 15-25$ (Wilson et al. 2001; Seljak 2002). Theoretical CDM predictions at these intermediate radii are not yet firm, but the best estimates give $\Upsilon_{B 5} \sim 15-20$ (e.g., Weinberg et al. 2004; Wright et al. 2004), somewhat higher than we infer for the PN galaxies.

Thus, it seems there is a population of elliptical galaxies largely bereft of dark matter. One explanation is that their primordial CDM halos have been stripped away or puffed up by interactions with other systems, as can happen in galaxy clusters (Natarajan et al. 2002). However, these ellipticals are not in such rich environments. An alternative is that this is another manifestation of the generic problem of insufficiently centrally concentrated dark matter; we infer halo concentrations $c \sim 5$ while CDM predicts $c \sim 15$ before including baryonic effects. This interpretation is supported by studies at smaller radii with gravitational lenses - where the dark matter fraction is lower than CDM 
expectations, especially given additional priors on $H_{0}$ (Rusin et al. 2003) - and with dynamical model fits to the fundamental plane (Borriello et al. 2003). Further observational and theoretical investigations are needed to see if this "missing missing mass" presents a major predicament for CDM.

\section{References}

Baes, M., \& Dejonghe, H. 2001, ApJ, 563, L19

Bahcall, N. A., Lubin, L. M., \& Dorman, V. 1995, ApJ, 447, L81

Beasley, M. A., et al. 2004, in preparation

Bendo, G. J., \& Barnes, J. E. 2000, MNRAS, 316, 315

Bergond, G., et al., in preparation

Bertin, G., et al. 1994, A\&A, 292, 381

Borriello, A., Salucci, P., \& Danese, L. 2003, MNRAS, 341, 1109

Ciardullo, R., et al. 1998, ApJ, 492, 62

Côté, P., et al. 2001, ApJ, 559, 828

Côté, P., et al. 2003, ApJ, 591, 850

Douglas, N. G., et al. 2002, PASP, 114, 1234

Eke, V., Efstathiou, G., \& Wright, L. 2000, MNRAS, 315, L18

Fall, S. M. 1983, in Internal Kinematics and Dynamics of Galaxies, ed. E. Athanassoula (Dordrecht: Reidel), 391

Gerhard, O., Kronawitter, A., Saglia, R. P., \& Bender, R. 2001, AJ, 121, 1936

Lambas, D. G., Maddox, S. J., \& Loveday, J. 1992, MNRAS, 258, 404

Loewenstein, M., \& White, R. E., III. 1999, ApJ, 518, 50

Matsushita, K., et al. 2002, A\&A, 386, 77

Méndez, R. H., et al. 2001, ApJ, 563, 135

Natarajan, P., Kneib, J.-P., \& Smail, I. 2002, ApJ, 580, L11

Navarro, J. F., Frenk, C. S., \& White, S. D. M. 1995, MNRAS, 275, 56

O'Sullivan, E., Ponman, T. J., \& Collins, R. S. 2003, MNRAS, 340, 1375

Peng, E. W., Ford, H. C., \& Freeman, K. C. 2002, in Extragalactic Star Clusters, ed. D. Geisler, E. K. Grebel, \& D. Minniti. (San Francisco: ASP), 312

Prada, F., et al. 2003, ApJ, in press (astro-ph/0301360)

Romanowsky, A. J., \& Kochanek, C. S. 2001, ApJ, 553, 722

Romanowsky, A. J., et al. 2003, Science, 301, 1696

Rusin, D., Kochanek, C. S., \& Keeton, C. R. 2003, ApJ, 595, 29

Seljak, U. 2002, MNRAS, 334, 797

Schindler, S., Binggeli, B., \& Böhringer, H. 1999, A\&A, 343, 420

Schneider, S. E., et al., 1989, AJ, 97, 666

Weil, M. L., \& Hernquist, L. 1996, ApJ, 460, 101

Weinberg, D. H., et al. 2004, ApJ, in press (astro-ph/0212356)

Wilson, G., Kaiser, N., Luppino, G. A., \& Cowie, L. L. 2001, ApJ, 555, 572

Wright, et al., 2004, MNRAS, submitted (astro-ph/0310513) 